\title{
Manejo químico de adubos verdes para sucessão da cana-de-açúcar em sistema de cultivo mínimo ${ }^{1}$
}

\author{
Chemical management of green manure for succession of sugar cane crop in \\ minimum tillage system
}

\author{
Antonio Mendes de Oliveira Neto ${ }^{2}$, Cleber Daniel de Goes Maciel $^{3}$, Naiara Guerra ${ }^{1}$, Gesley \\ Guimarães de Ramos Lima ${ }^{4}$, Luis Carlos Sola Júnior ${ }^{3}$
}

\begin{abstract}
Resumo - Estudos referentes a manejo químico de adubos verdes são escassos na literatura nacional. O trabalho teve com objetivo avaliar a eficiência de herbicidas no controle químico dos adubos verdes para sucessão com a cultura da cana-de-açúcar, em sistema de cultivo mínimo. Foram conduzidos dois ensaios com as espécies Crotalaria spectabilis e Mucuna aterrima em delineamento de blocos ao acaso com 19 tratamentos e quatro repetições. Os tratamentos foram constituídos de três dosagens distintas para os herbicidas: glyphosate + 2,4-D + carfentrazoneethyl; glyphosate + 2,4-D; glyphosate + MSMA; 2,4-D + carfentrazone; 2,4-D; 2,4-D + picloran e uma testemunha sem aplicação. O controle químico com as misturas em tanque de glyphosate + 2,4 -D + carfentrazone-ethyl e glyphosate $+2,4$-D foram às alternativas com maior sucesso no manejo de $M$. aterrima e $C$. spectabilis, as quais podem viabilizar a possibilidade de sucessão do plantio da cana-de-açúcar em sistema de cultivo mínimo, com economia das operações de aração e gradagem.
\end{abstract}

Palavras-chave: Dessecação; Crotalaria spectabilis; Mucuna aterrima.

\begin{abstract}
Studies on chemical management of green manure are scarce in national literature. This work aimed to evaluate the herbicides efficiency in chemical control of green manure used in succession with sugarcane crop, in minimum cultivation system. Two experiments were conducted with Mucuna aterrima and Crotalaria spectabilis species, arranged in a randomized complete block design with 19 treatments and four replicates. The treatments were composed by three distinct rate for herbicides: glyphosate+2,4-D+carfentrazone-ethyl; glyphosate+2,4-D; glyphosate+MSMA; 2,4-D+carfentrazone-ethyl; 2,4-D; 2,4-D+picloran and check without herbicide application. Chemical control with tank mixture between glyphosate+2,4D+carfentrazone-ethyl and glyphosate+2,4-D were the outstanding alternatives on of M. aterrima and $C$. spectabilis management, allowing the possibility of succession planting sugar cane crop in minimum cultivation system, with economy of operations of plowing and harrowing.

Keywords: Burndown; Crotalaria spectabilis; Mucuna aterrima.

\footnotetext{
${ }^{1}$ Recebido da publicação em 11/04/2011 e aceito em 30/07/2011.

${ }^{2}$ Discente do Programa de Pós-Graduação em Agronomia da Universidade Estadual de Maringá (UEM/NAPD), Av. Colombo 5790, 87020-900, Maringá, PR. E-mail: am.oliveiraneto@gmail.com; naiara.guerra@ hotmail.com.

3 Eng ${ }^{\circ}$-Agro ${ }^{\circ}$, D.Sc. Professor Adjunto, Depto de Ciências Agronômicas - Universidade Estadual de Maringá, UEM. Campus Avançado de Umuarama/PR - CAU/CCA. Estrada da Paca s/n, 87507-190, Umuarama/PR E-mail: cdgmaciel2@uem.br

${ }^{4}$ Discente do curso de Agronomia da Escola Superior de Agronomia de Paraguaçu Paulista, ESAPP. E mail: gesley.lima@hotmail.com; kakasolajr@hotmail.com
} 


\section{Introdução}

A adubação verde é uma prática conhecida desde a antiguidade, podendo ser definida como a incorporação ao solo de material vegetal não decomposto, produzido ou não no local, a qual resulta em melhoria desejável das características químicas, físicas e biológicas do solo (Cáceres \& Alçardes, 1995; Wutke \& Arévalo, 2006).

A Crotalaria spectabilis e Mucuna aterrima produzem, respectivamente, em torno de 4,2 e 3,0 toneladas de matéria seca por hectare (Cáceres \& Alçardes, 1995; Garcia, 2002; Nascimento \& Silva, 2004), e juntamente com outras espécies de adubo verde como $C$. juncea, Lablab purpureus, Arachis hypogaea e Glycine max têm sido utilizadas em sucessão na reforma do canavial, devido a rápida decomposição favorecer a mineralização e disponibilização de nitrogênio à cana-de-açúcar (Ambrosano et al. 2005; Wutke \& Arévalo, 2006). Este procedimento pode incrementar a produtividade de colmos devido à fixação de nitrogênio, aumento da capacidade de trocas de cátions, controle de plantas daninhas e reciclagem de nutrientes (Espanhol et al., 2007a,b).

O emprego de adubos verdes pode ser uma técnica eficiente no controle de plantas daninhas, principalmente devido a efeitos físicos e alelopáticos. Fontanétti et al. (2004), observaram que as espécies mucuna-preta (Estizolobium aterrimum) e feijão-de-porco (Canavalia ensiformes) reduziram significativamente o número de plantas de tiririca por metro quadrado quando incorporadas ao solo antes do plantio de alface-americana e repolho. Já plantas como $C$. juncea, $C$. spectabilis, $M$. aterrima e $M$. pruriensis são plantas que possuem alta capacidade competitiva reduzindo a produção de massa seca e o número de plantas daninhas (Fernandes et al., 1999; Erasmo et al., 2004; Cava et al., 2008), além da possibilidade de serem utilizadas na fitorremediação de solos contaminados com herbicidas (Procópio et al., 2005; Pires et al., 2006).

Severino \& Christoffoleti (2001), constataram que a utilização dos adubos verdes Arachis pintoi, C. juncea e Cajanus cajan reduziram significativamente o banco de sementes das espécies Brachiaria decumbens, Panicum maximum e Bidens pilosa.

A dessecação é uma das etapas mais importantes para qualquer época do ano e cultura, pois a aplicação de herbicidas nãoseletivos na eliminação da cobertura vegetal assegura a perfeita emergência da cultura, permitindo o crescimento inicial sem interferência de plantas daninhas (Christoffoleti et al., 2005). Portanto, o manejo químico de adubos verdes para sucessão da cultura da cana-de-açúcar pode ser uma ferramenta importante e viável no sistema de cultivo mínimo, pois, a adequada dessecação da cobertura vegetal facilita sobremaneira as operações de implantação de um novo canavial, podendo dispensar operações como a aração e gradagens para incorporação dos adubos verdes.

O trabalho teve como objetivo avaliar a eficiência de herbicidas no manejo químico dos adubos verdes Crotalaria spectabilis e Mucuna aterrima, utilizados em sucessão com a cultura da cana-de-açúcar na forma de cultivo mínimo.

\section{Material e métodos}

Dois experimentos foram conduzidos durante os meses de Janeiro e Fevereiro de 2008, em áreas com solo de textura argilosa, pertencentes à Fazenda Rancho Alegre, localizada no Município de Paraguaçu Paulista/SP. O primeiro experimento foi semeado a lanço $80 \mathrm{~kg}$ de sementes por hectare do adubo verde $C$. spectabilis no dia 27/10/2007, em talhão localizado a $22^{\circ} 32^{\prime} 08,05^{\prime \prime}$ de latitude Sul, 50³6'32,10” de longitude Oeste e 426 metros de altitude. No 
segundo, a semeadura também foi realizada a lanço em 01/11/2007, utilizando-se $15 \mathrm{~kg}$ de sementes de $M$. aterrima por hectare, em talhão localizado a $22^{\circ} 30^{\prime} 45,67^{\prime}$ ' de latitude sul, 50 $37^{\prime} 19,25^{\prime \prime}$ de longitude oeste e 456 metros de altitude. Em ambos os experimentos foi programado o plantio da cultura da canade-açúcar em sucessão aos adubos verde aos 30 dias após o procedimento de dessecação com herbicidas. Dessa forma, utilizou-se no preparo do solo com sulcamento das linhas sobre os resíduos das plantas dessecadas, de forma a suprimir as operações de aração e gradagem, caracterizando um sistema de cultivo mínimo.

Nos experimentos foram avaliados 19 tratamentos herbicidas constituídos por diferentes associações e dosagens (Tabela 1), dispostos em delineamento de blocos ao acaso (DBC) com quatro repetições. As unidades experimentais apresentaram área total de 21 $\mathrm{m}^{2}(3,0 \times 7,0 \mathrm{~m})$, e para efeito das avaliações desprezou-se $0,5 \mathrm{~m}$ das laterais e extremidades de cada parcela, conferindo as mesmas, áreas úteis de $12 \mathrm{~m}^{2}$.

Tabela 1. Descrição dos herbicidas e doses utilizados no manejo químico de $M$. aterrima e $C$. spectabilis, Paraguaçu Paulista /SP, 2008.

\begin{tabular}{|c|c|c|}
\hline \multirow{2}{*}{ Tratamentos } & M. aterrima & C. spectabilis \\
\hline & Dosagem g i.a. ou e.a. ha $^{-1}$ & Dosagem g i.a. ou e.a. ha $^{-1}$ \\
\hline T1- glyphosate+2,4-D+carfentrazone & $1440+2010+8$ & $2160+3350+16$ \\
\hline T2- glyphosate+2,4-D+carfentra & $2160+1340+8$ & $2880+2680+16$ \\
\hline T3- glyphosate $+2,4-\mathrm{D}+$ carfentrazone & $2880+670+8$ & $3600+2010+16$ \\
\hline T4- glyphosate+2,4-D & $1440+2010$ & $2160+3350$ \\
\hline T5- glyphosate+2,4-D & $2160+1340$ & $2880+2680$ \\
\hline T6- glyphosate+2,4-D & $2880+670$ & $3600+2010$ \\
\hline T7- glyphosate+MSMA & $1 \overline{440}+2 \overline{3} \overline{7} 0$ & $2 \overline{1} \overline{6} \overline{0}+31 \overline{16}-\overline{0}$ \\
\hline T8- glyphosate+MSMA & $2160+1580$ & $2880+2370$ \\
\hline T9- glyphosate+MSMA & $2880+790$ & $3600+1580$ \\
\hline T10-2,4-D+carfentrazone & $2010+8$ & $2010+1 \overline{6}$ \\
\hline T11-2,4-D+carfentrazone & $1340+8$ & $2680+16$ \\
\hline T12-2,4-D+carfentrazone & $670+8$ & $3350+16$ \\
\hline $\mathrm{T} 13-\overline{4}-\overline{4}-\overline{\mathrm{D}}$ & 2010 & $20 \overline{10}$ \\
\hline T14- 2,4-D & 1340 & 2680 \\
\hline T15-2,4-D & 670 & 3350 \\
\hline T16- $2, \overline{4}-\mathrm{D}+$ picloran & $120+32$ & $\overline{360+96}$ \\
\hline T17- 2,4-D+picloran & $240+64$ & $480+128$ \\
\hline T18- 2,4-D+picloran & $480+128$ & $720+192$ \\
\hline T19-Testemunhà & & \\
\hline
\end{tabular}

Obs.: Em todos os tratamentos herbicidas foi adicionado $0,25 \% \mathrm{v} \mathrm{v}^{-1}$ do Adjuvante Joint Oil. 
A pulverização dos tratamentos foi realizada em 24/01/2008 com auxílio de um pulverizador costal pressurizado constantemente com $\mathrm{CO}_{2}$ a $207 \mathrm{kPa}$, e munido com barra de seis pontas DG $11002 \mathrm{VS}$ espaçadas em $50 \mathrm{~cm}$ e localizadas a $50 \mathrm{~cm}$ de altura dos adubos verdes, proporcionando o consumo de calda de $200 \mathrm{~L} \mathrm{ha}^{-1}$. No momento da aplicação os adubos verdes encontravam-se fase vegetativa com apenas algumas poucas plantas em início de floração. As condições climáticas médias foram de ventos de $2,0 \mathrm{~km}$ $\mathrm{h}^{-1}$, temperatura $28,2^{\circ} \mathrm{C}$ e umidade relativa do ar, em torno de $70 \%$. Os dados meteorológicos referentes às médias semanais de temperatura $\left({ }^{\circ} \mathrm{C}\right)$ e pluviometria $(\mathrm{mm})$ encontram-se dispostos na Figura 1.

Avaliou-se o percentual de controle dos adubos verdes aos 3, 7, 14, 21 e 28 dias após a aplicação (DAA) dos tratamentos, utilizando escala de nota de 0 a $100 \%$, onde a nota 0 corresponde a ausência de controle, e a nota 100, a morte total das plantas (SBCPD, 1995).

Os dados obtidos foram submetidos à análise de variância pelo teste $\mathrm{F}$ e as suas médias comparadas pelo teste de agrupamento de Scott-Knott (1974), ao nível de $5 \%$ de probabilidade.

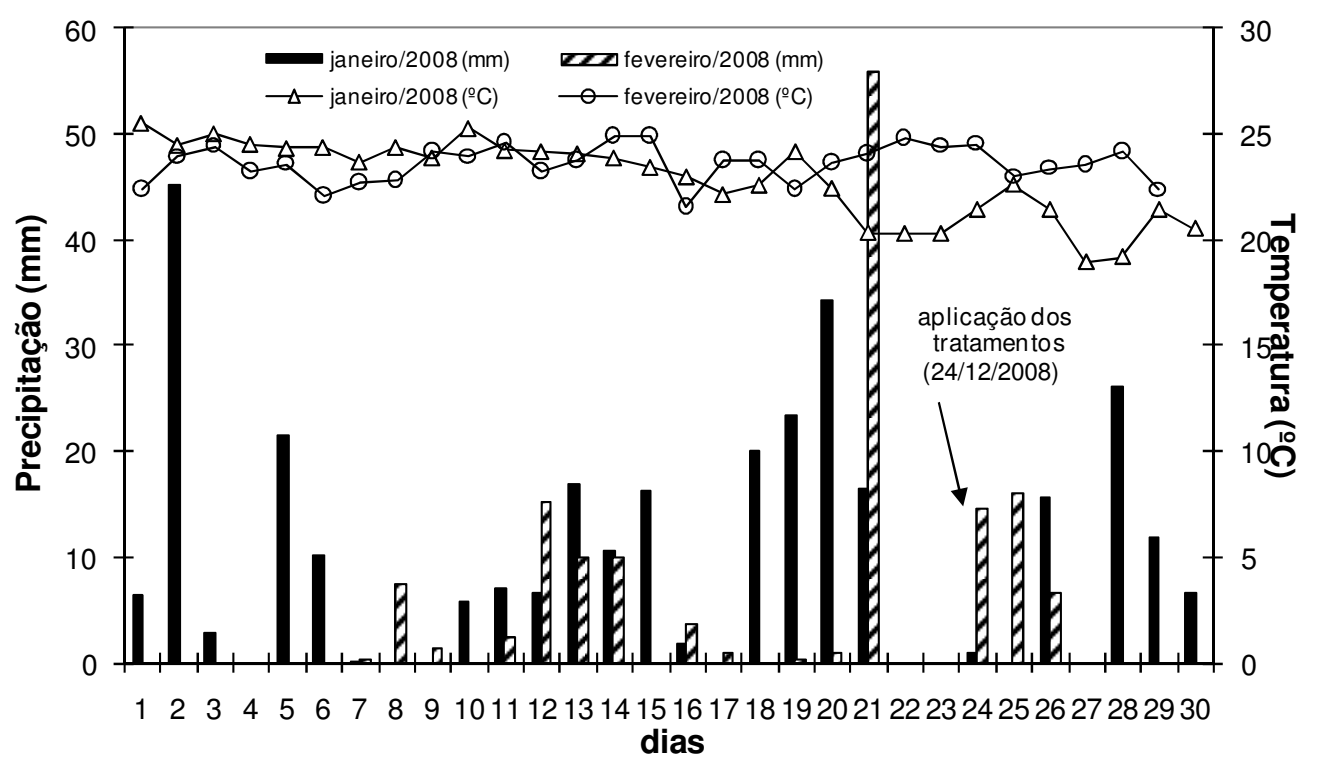

Figura 1. Dados climáticos referentes ao período de condução dos experimentos. 


\section{Resultados e discussão}

$\mathrm{Na}$ primeira avaliação realizada aos 3 DAA, as misturas em tanque de glyphosate + 2,4-D + carfentrazone, glyphosate + 2,4-D e glyphosate + MSMA, em todas as dosagens, apresentaram níveis de controle de $M$. aterrima de $68,8 \%$ e $81,3 \%$, e destacando-se como agrupamento de tratamentos de maior precocidade na dessecação destas espécie (Tabela 2).

A partir de 7 DAA, a associação entre os herbicidas glyphosate + 2,4-D + carfentrazone, glyphosate $+2,4$-D e glyphosate + MSMA, apresentaram excelente controle de M. aterrima $(>90,0 \%)$ independentemente da dosagens avaliadas, de forma que a parte aérea das plantas apresentavam-se com elevado grau de dessecação. Nessa avaliação, os tratamentos onde foram utilizados 2,4-D + carfentazone, 2,4-D e 2,4-D + picloran apresentaram controle insatisfatório. Essa ação mais lenta para os tratamentos a base de herbicidas com mecanismo de ação de mimetizadores de auxina é comum em suas recomendações, as quais normalmente mencionam de 3 a 5 semanas para expressarem o seu efeito na totalidade sobre as plantas daninhas (Oliveira Jr., 2001; Rodrigues \& Almeida, 2005).

Aos 14 DAA, as associações entre glyphosate + 2,4-D + carfentrazone e glyphosate $+2,4-\mathrm{D}$, promoveram a máxima eficácia de controle de M. aterrima (100,0\%), e juntamente com glyphosate + MSMA e 2,4-D + picloran, nas dosagens de $2880+790$ e $480+$
$128 \mathrm{~g} \mathrm{ha}^{-1}$, formando o grupo dos melhores tratamentos herbicidas, segundo os critérios de Scott \& Knott. Os tratamentos glyphosate + MSMA $\left(1440+2370\right.$ e $\left.2160+1580 \mathrm{~g} \mathrm{ha}^{-1}\right)$, 2,4-D + picloran $\left(240+64 \mathrm{~g} \mathrm{ha}^{-1}\right)$ e $2,4-\mathrm{D}+$ carfentrazone $\left(2010+8 \mathrm{~g} \mathrm{ha}^{-1}\right)$ também apresentaram controle eficiente de $M$. aterrima (> 90,0\%), mas caracterizam-se pela formação de um grupo secundário aos 14 DAA. Com exceção de 2,4-D e 2,4-D + picloran, nas respectivas dosagens de 670 e $120+32 \mathrm{~g} \mathrm{ha}^{-1}$, todos os demais tratamentos apresentaram controle satisfatório de M. aterrima $(>80,0 \%)$ aos 14 DAA.

Nas últimas avaliações, realizadas aos 21 e 28 DAA (Tabela 2), todos os tratamentos apresentaram excelente controle de $M$. aterrima, de forma que a porcentagem mínima de controle foi da ordem de $91,5 \%$. No entanto, as misturas de glyphosate + 2,4-D + carfentrazone, glyphosate $+2,4-\mathrm{D}$ e glyphosate + MSMA, continuaram a merecer destaque por apresentarem níveis máximos de eficácia de controle $(100,0 \%)$, mesmo quando foram utilizados nas menores dosagens avaliadas, não sendo evidenciado falhas na dessecação das plantas e/ou mesmo à possibilidade de origem de novas rebrotas do adubo verde. Os resultados corroboram com os observados por Beckie et al. (2004) em estudo sobre doseresposta do herbicida 2,4-D, onde cultivares de Brassica napus mesmo em diferentes estádios de desenvolvimento, também mostraram-se bastante sensíveis a este herbicida. 
Tabela 2. Porcentagem de controle de $M$. aterrima aos 3, 7, 14, 21 e 28 dias após a aplicação (DAA) dos tratamentos, Paraguaçu Paulista/SP, 2008.

\begin{tabular}{|c|c|c|c|c|c|}
\hline \multirow{2}{*}{ Tratamentos (g ha-1) } & \multicolumn{5}{|c|}{ Controle $(\%)$} \\
\hline & 3 DAA & 7 DAA & 14 DAA & $21 \mathrm{DAA}$ & $28 \mathrm{DAA}$ \\
\hline 1. glypho+2,4-D+carfen $(1440+2010+8)$ & $70,8 \mathrm{~b}$ & 94,5 a & $100,0 \mathrm{a}$ & $100,0 \mathrm{a}$ & $100,0 \mathrm{a}$ \\
\hline 2. glypho+2,4-D+carfen ${ }^{(2160+1340+8)}$ & $73,3 \mathrm{~b}$ & 94,5 a & $100,0 \mathrm{a}$ & 100,0 a & 100,0 a \\
\hline 3. glypho+2,4-D+carfen ${ }^{(2880+670+8)}$ & $72,8 \mathrm{~b}$ & 97,8 a & $100,0 \mathrm{a}$ & 100,0 a & $100,0 \mathrm{a}$ \\
\hline 4. glyphosate+2,4-D $(1440+2010)$ & $70,8 \mathrm{~b}$ & 95,0 a & $100,0 \mathrm{a}$ & $100,0 \mathrm{a}$ & 100,0 a \\
\hline 5. glyphosate+2,4-D ${ }^{(2160+1340)}$ & $69,5 \mathrm{~b}$ & 95,5 a & 100,0 a & 100,0 a & 100,0 a \\
\hline 6. glyphosate+2,4-D ${ }^{(2880+670)}$ & $71,8 \mathrm{~b}$ & 98,3 a & 100,0 a & 100,0 a & 100,0 a \\
\hline 7. glyphosate+MSMA & 81,3 a & 94,0 a & $95,8 \mathrm{~b}$ & $98,3 \mathrm{~b}$ & 100,0 a \\
\hline 8. glyphosate+MSMA & $73,8 \mathrm{~b}$ & $90,3 \mathrm{~b}$ & $96,3 \mathrm{~b}$ & $98,5 \mathrm{~b}$ & 100,0 a \\
\hline 9. glyphosate+MSMA & $68,8 \mathrm{~b}$ & $91,0 \mathrm{~b}$ & 98,0 a & 100,0 a & 100,0 a \\
\hline 10. 2,4-D+carfentrazone ${ }^{(2010+8)}$ & $33,8 \mathrm{c}$ & $45,5 \mathrm{c}$ & $90,8 \mathrm{c}$ & $97,8 \mathrm{~b}$ & $98,0 \mathrm{~b}$ \\
\hline 11. 2,4-D+carfentrazone ${ }^{(1340+8)}$ & $30,0 \mathrm{c}$ & $39,3 \mathrm{~d}$ & $86,8 \mathrm{~d}$ & $92,3 \mathrm{~d}$ & $94,5 \mathrm{c}$ \\
\hline 12. 2,4-D+carfentrazone ${ }^{(670+8)}$ & $28,8 \mathrm{c}$ & $30,8 \mathrm{~d}$ & 80,3 e & $91,5 \mathrm{~d}$ & $92,3 \mathrm{~d}$ \\
\hline 13. $2,4-\mathrm{D}^{(2010)}$ & $27,5 \mathrm{c}$ & $39,5 \mathrm{~d}$ & $86,0 \mathrm{~d}$ & $97,0 \mathrm{~b}$ & $97,3 \mathrm{~b}$ \\
\hline 14. $2,4-\mathrm{D}^{(1340)}$ & $23,0 \mathrm{~d}$ & 34,5 e & 82,5 e & $94,0 \mathrm{c}$ & $95,3 \mathrm{c}$ \\
\hline 15. $2,4-\mathrm{D}^{(670)}$ & $21,3 \mathrm{~d}$ & 33,3 e & $75,8 \mathrm{f}$ & $94,3 \mathrm{c}$ & $94,8 \mathrm{c}$ \\
\hline 16. $2,4-\mathrm{D}+$ picloran $^{(120+32)}$ & $22,8 \mathrm{~d}$ & 30,8 e & $69,0 \mathrm{~g}$ & $92,3 \mathrm{~d}$ & $94,0 \mathrm{c}$ \\
\hline 17. 2,4-D+picloran $(240+64)$ & $21,3 \mathrm{~d}$ & $38,5 \mathrm{~d}$ & $96,0 \mathrm{~b}$ & $98,5 \mathrm{~b}$ & $98,3 \mathrm{~b}$ \\
\hline 18. 2,4-D+picloran ${ }^{(480+128)}$ & $27,5 \mathrm{c}$ & 56,5 e & 99,0 a & $100,0 \mathrm{a}$ & 99,8 a \\
\hline 19. testemunha & $0,0 \mathrm{e}$ & $0,0 \mathrm{f}$ & $0,0 \mathrm{~h}$ & $0,0 \mathrm{e}$ & $0,0 \mathrm{e}$ \\
\hline $\bar{F}$ & $229,38^{*}$ & $486,24^{*}$ & $297,25^{*}$ & $767,26^{*}$ & $21,09^{*}$ \\
\hline $\mathrm{CV}(\%)$ & 7,36 & 4,6 & 3,08 & 1,76 & 1,06 \\
\hline
\end{tabular}

- Médias seguidas de mesma letra minúscula na coluna, pertencem ao mesmo agrupamento de Scott-Knott, a 5\% de probabilidade. $*$ = significativo; NS = não significativo.

De forma geral, a evolução do controle ocorreu de forma mais lenta para o adubo verde $C$. spectabilis em relação aos resultados observado com $M$. aterrima. Aos 3 DAA, todos os tratamentos herbicidas apresentaram baixo nível de eficácia para $C$. spectabilis, de forma que o melhor tratamento não superou $40,0 \%$ de controle (Tabela 3 ).

$\mathrm{Na}$ avaliação subseqüente, realizada aos 7 DAA, houve apenas uma pequena evolução nos níveis de controle de $C$. spectabilis, a qual seguiu a mesma tendência da avaliação aos 3 DAA, onde o agrupamento de tratamentos que se destacaram foram glyphosate $+2,4-\mathrm{D}+$ carfentrazone, glyphosate + 2,4-D e glyphosate + MSMA, nas três dosagens avaliadas. Os tratamentos constituídos por 2,4-D + carfentrazone, 2,4-D e 2,4-D + picloran, diferiram significativamente dos demais tratamentos, sendo também agrupados pelo critério de Scott \& Knott, mas pela menor média de controle $(<17,0 \%)$. Aos 14 DAA, glyphosate + 2,4-D + carfentrazone, glyphosate $+2,4-\mathrm{D}$ e glyphosate + MSMA, na dosagem $2880+$ $2370 \mathrm{~g} \mathrm{ha}^{-1}$, melhoraram significativamente o controle de $C$. spectabilis para patamares aceitáveis, ou seja, superior a 80,0\%, formando o melhor agrupamento do período.

Nas avaliações aos 21 e 28 DAA (Tabela 3), de forma semelhante à constatada para $M$. aterrima, todos os tratamentos com glyphosate $+2,4-\mathrm{D}+$ carfentrazone e glyphosate $+2,4-\mathrm{D}$, assim como de glyphosate 
+ MSMA, em $3600+1580 \mathrm{~g} \mathrm{ha}^{-1}$, superaram significativamente os demais, com excelentes níveis de controle de $C$. spectabilis $(>90,0 \%)$, formando um agrupamento com excelente eficácia de controle. Aos 28 DAA, também se destacaram pelo controle satisfatório de $C$. spectabilis $(>80,0 \%)$ a associação de $2,4-\mathrm{D}+$ carfentrazone, nas dosagens de $2010+16$ e
$2680+16 \mathrm{~g} \mathrm{ha}^{-1}$, e glyphosate + MSMA, com $2880+2370 \mathrm{~g} \mathrm{ha}^{-1}$. Os demais tratamentos promoveram níveis de controle de $C$. spectabilis insatisfatório aos 28 DAA, ou seja, inferiores a $80,0 \%$.

Tabela 3. Porcentagem de controle de C. spectabilis aos 3, 7, 14, 21 e 28 dias após a aplicação (DAA) dos tratamentos, Paraguaçu Paulista/SP, 2008.

\begin{tabular}{|c|c|c|c|c|c|}
\hline \multirow{2}{*}{ Tratamentos (g ha-1) } & \multicolumn{5}{|c|}{ Controle $(\%)$} \\
\hline & 3 DAA & 7 DAA & 14 DAA & $21 \mathrm{DAA}$ & 28 DAA \\
\hline 1. glypho+2,4-D+carfen ${ }^{(2160+3350+16)}$ & 39,5 a & $47,3 \mathrm{a}$ & 82,0 a & 93,3 a & 97,3 a \\
\hline 2. glypho+2,4-D+carfen ${ }^{(2880+2680+16)}$ & 36,5 a & 47,3 a & 83,0 a & $89,5 \mathrm{~b}$ & 96,8 a \\
\hline 3. glypho+2,4-D+carfen ${ }^{(3600+2010+16)}$ & 37,3 a & $47,5 \mathrm{a}$ & 85,8 a & 96,3 a & 98,8 a \\
\hline 4. glyphosate+2,4-D ${ }^{(2160+3350)}$ & 37,5 a & 43,8 a & 81,8 a & 94,8 a & 97,8 a \\
\hline 5. glyphosate $+2,4-\mathrm{D}^{(2880+2680)}$ & $32,5 \mathrm{~b}$ & 45,5 a & 83,5 a & 98,0 a & 97,5 a \\
\hline 6. glyphosate $+2,4-\mathrm{D}^{(3600+2010)}$ & $34,3 \mathrm{~b}$ & 42,8 a & 82,5 a & 97,8 a & 97,8 a \\
\hline 7. glyphosate+MSMA ${ }^{(2160+3160)}$ & 36,3 a & 48,8 a & $64,3 \mathrm{c}$ & $67,0 \mathrm{~d}$ & $69,5 \mathrm{c}$ \\
\hline 8. glyphosate+MSMA & 36,3 a & $47,0 \mathrm{a}$ & $69,3 \mathrm{~b}$ & $83,3 \mathrm{c}$ & $84,3 \mathrm{~b}$ \\
\hline 9. glyphosate+MSMA ${ }^{(3600+1580)}$ & 36,0 a & $46,5 \mathrm{a}$ & 85,0 a & 96,5 a & 97,5 a \\
\hline 10. 2,4-D+carfentrazone ${ }^{(2010+16)}$ & $16,3 \mathrm{c}$ & $16,5 \mathrm{~b}$ & $24,0 \mathrm{~d}$ & $47,5 \mathrm{e}$ & $84,5 \mathrm{~b}$ \\
\hline 11. 2,4-D+carfentrazone ${ }^{(2680+16)}$ & $15,8 \mathrm{c}$ & $16,0 \mathrm{~b}$ & $22,5 \mathrm{~d}$ & 50,0 e & $84,5 b$ \\
\hline 12. $2,4-\mathrm{D}+$ carfentrazone $^{(3350+16)}$ & $16,8 \mathrm{c}$ & $15,5 \mathrm{~b}$ & $24,3 \mathrm{~d}$ & 51,3 e & 94,3 a \\
\hline 13. 2,4-D & $10,5 \mathrm{~d}$ & $12,8 \mathrm{~b}$ & $15,8 \mathrm{e}$ & $25,0 \mathrm{~g}$ & $40,8 \mathrm{e}$ \\
\hline 14. 2,4-D & $14,5 \mathrm{c}$ & $15,0 \mathrm{~b}$ & $16,5 \mathrm{e}$ & $25,8 \mathrm{~g}$ & $48,8 \mathrm{~d}$ \\
\hline $15.2,4-\mathrm{D}^{(3350)}$ & $14,5 \mathrm{c}$ & $15,0 \mathrm{~b}$ & $19,5 \mathrm{e}$ & $37,5 \mathrm{f}$ & $71,5 \mathrm{c}$ \\
\hline 16. 2,4-D+picloran ${ }^{(360+96)}$ & $11,3 \mathrm{~d}$ & $13,8 \mathrm{~b}$ & $20,8 \mathrm{~d}$ & $36,5 \mathrm{f}$ & $70,0 \mathrm{c}$ \\
\hline 17. 2,4-D+picloran ${ }^{(480+128)}$ & $12,0 \mathrm{~d}$ & $12,8 \mathrm{~b}$ & $21,3 \mathrm{~d}$ & 48,8 e & $74,5 \mathrm{c}$ \\
\hline 18. 2,4-D+picloran ${ }^{(720+192)}$ & $12,0 \mathrm{~d}$ & $15,0 \mathrm{~b}$ & $24,5 \mathrm{~d}$ & $87,5 \mathrm{~b}$ & 96,0 a \\
\hline 19. testemunha & $0,0 \mathrm{e}$ & $0,0 \mathrm{c}$ & $0,0 \mathrm{f}$ & $0,0 \mathrm{~h}$ & $0,0 \mathrm{f}$ \\
\hline $\bar{F}$ & $140,87^{*}$ & $122,51^{*}$ & $449,17^{*}$ & $570,21^{*}$ & $154,42^{*}$ \\
\hline $\mathrm{CV}(\%)$ & 9,11 & 10,9 & 6,33 & 4,01 & 5,24 \\
\hline
\end{tabular}

- Médias seguidas de mesma letra minúscula na coluna, pertencem ao mesmo agrupamento de Scott-Knott, a 5\% de probabilidade. ${ }^{*}=$ significativo; $\mathrm{NS}=$ não significativo.

Esses resultados corroboram com os obtidos por Espanhol et al. (2007a,b), que relataram níveis de controles superiores a $80,0 \%$ para o adubo verde Crotalaria juncea aos 28 DAA, utilizando glyphosate + metsulfuron-methyl $\left(1620+8 \mathrm{~g} \mathrm{ha}^{-1}\right) \mathrm{e}$ glyphosate + 2,4-D $\left(1620+1209 \mathrm{~g} \mathrm{ha}^{-1}\right)$. No entanto, os autores constataram a ocorrência de um elevado número de rebrota das plantas dessecadas, devido à quebra da dominância da gema apical.

Dentre as espécies avaliadas, foi possível constatar que a $C$. spectalis apresentou maior dificuldade de ser manejada com os herbicidas estudados quando comparada com $M$. aterrima, apesar da $M$. aterrima possuir maior quantidade de massa verde da parte aérea no momento da aplicação. 
Entretanto, as misturas em tanque com glyphosate $+2,4-\mathrm{D}$, glyphosate + 2,4-D + carfentazone, glyphosate + MSMA $(2880+$ 2370 e $\left.3600+1580 \mathrm{~g} \mathrm{ha}^{-1}\right), 2,4-\mathrm{D}+$ carfentrazone e 2,4-D + picloran $(720+192 \mathrm{~g}$ $\mathrm{ha}^{-1}$ ) mostraram-se promissoras para o manejo químico das duas espécies de adubos verde.

Os resultados obtidos indicaram a viabilidade do controle químico de adubos verdes $M$. aterrima $e C$. spectalis na reforma do canavial. Diferentemente da testemunha não manejada, aos 30 dias após dessecação, todos os tratamentos herbicidas estudados, mesmo aqueles que controlaram insatisfatoriamente a $C$. spectabilis aos 28 DAA, ainda assim possibilitaram realizar com sucesso o sulcamento e plantio da cana-deaçúcar, em sistema de cultivo mínimo. Além do aspecto de economia das operações de aração e gradagem, também é importante considerar, que em função das características dos herbicidas estudados, em todos os tratamentos onde se utilizou glyphosate não foi constatada falhas no controle de algumas espécies de plantas daninhas gramíneas, como as do gênero Digitaria e Brachiaria (dados não demonstrados). Nesse sentido, a realização de novos trabalhos, visando avaliar a eficiência de doses-resposta de outros herbicidas e associações como manejo alternativo, assim como de outras espécies de adubos verdes são de fundamental importância para maior acertabilidade do sistema de cultivo mínimo na cultura da cana-de-açúcar.

\section{Conclusões}

Nas condições em que foram conduzidos os experimentos conclui-se que o controle químico com as misturas em tanque de glyphosate $+2,4-\mathrm{D}+$ carfentrazone-ethyl e glyphosate $+2,4-\mathrm{D}$ foram às alternativas com maior sucesso no manejo de Mucuna aterrima e Crotalaria spectabilis, viabilizando a sucessão do plantio da cana-de-açúcar em sistema de cultivo mínimo, com economia das operações de aração e gradagem.

\section{Referências}

AMBROSANO, E.A. et al. Utilization of nitrogen fron gren manure and mineral fertilizer by sugarcane. Scientia Agrícola, v.62, n3, p.534-542, 2005.

BECKIE, H.J. et al. Multiple herbicideresistant canola can be controlled by alternative herbicides. Weed Science, v.52, p.152-157, 2004.

CÁCERES, N.T.; ALCARDE, J.C. Adubação verde com leguminosas em rotação com a cultura da cana-de-açúcar (Saccharum sp.). STAB açúcar, álcool e subprodutos, v.13, n.5, p.16-20, 1995.

CAVA, M.G.B. et al. Adubos verdes para a renovação de canaviais do sudoeste goiano. In: CONGRESSO INTERNACIONAL DE TECNOLOGIA NA CADEIA PRODUTIVA DA CANA, 2, 2008, Uberaba. Anais... Uberaba, 2008. (CD-ROM)

CHRISTOFFOLETI, P.J.; CARVALHO, S.J.P.; NICOLAI, M. Hora certa. Cultivar. 2007. Disponível em: $<$ http://www.cultivar.inf.br> Acesso em: 27 ago. 2008.

ESPANHOL, M. et al. Dessecação de Crotalaria juncea no outono/inverno com glyphosate e 2,4-D.In: SIMPÓSIO INTERNACIONAL SOBRE GLYPHOSATE, 2007, Botucatu. Anais... Londrina: UNESP, 2007. p. 124-127.

ESPANHOL, M. et al. Dessecação de Crotalaria juncea no outono/inverno com glyphosate e metsulfuron-methyl. In: SIMPÓSIO INTERNACIONAL SOBRE GLYPHOSATE, 2007, Botucatu. Anais... Londrina: UNESP, 2007. p. 128-131. 
ERASMO, E.A.L. et al. Potencial de espécies utilizadas como adubo verde no manejo integrado de plantas daninhas. Planta Daninha, v.22, n.3, p.337-342, 2004.

FERNANDES, M. F.; BARRETO, A.C.; EMÍDIO FILHO, J. Fitomassa de adubos verdes e controle de plantas daninhas em diferentes densidades populacionais de leguminosas. Pesquisa Agropecuária Brasileira, v.34, n.9, p.1593-1600, 1999.

FONTANÉTTI, A. et al. Adubação verde no controle de plantas invasoras nas culturas de alface-americana e de repolho. Ciência e Agrotecnologia, v.28, n.5, p.967-973, 2004.

GARCIA, L. F. Introdução e avaliação de leguminosas para adubação verde em solos arenosos de tabuleiros costeiros do Piauí. Revista Faculdade de Agronomia, v.28, n.2, p.93-103, 2002.

NASCIMENTO, J. T.; SILVA, I. F. Avaliação quantitativa e qualitativa da fitomassa de leguminosas para o uso como cobertura do solo. Ciência Rural, v.34, n.3, p.947-949, 2004.

OLIVEIRA JR., R.S. Mecanismo de ação de herbicidas. In: OLIVEIRA JR., R.S.; CONSTANTIN, J. (Coord.). Plantas daninhas e seu manejo. Guaíba: Agropecuária, 2001, p.209-260.

PIRES, F.R. et al. Adubos verdes na fitorremediação de solos contaminados com o herbicida tebuthiuron. Caatinga, v.19, n.1, p.92-97, 2006.

PROCÓPIO, S.O. et al. Potencial de espécies vegetais para remediação do herbicida tryfloxysulfuron-sodium. Planta Daninha, v.23, n.1, p.9-16, 2005.

RODRIGUES, B.N.; ALMEIDA, F.S. Guia de herbicidas. $5^{\text {a }}$ edição. Londrina: Editora dos autores. 2005. 592p.
SEVERINO, F. J.; CHRISTOFFOLETI, P. J. Banco de sementes de plantas daninhas em solo cultivado com adubos verdes. Bragantia, v.60, n.3, p.201-204, 2001.

SOCIEDADE BRASILEIRA DA CIÊNCIA
DAS PLANTAS

Procedimentos para instalação, avaliação e análise de experimentos com herbicidas. Londrina: SBCPD, 1995. 42p.

SCOTT, A.; KNOTT, M. Cluster-analysis method for grouping means in analysis of variance. Biometrics, v.30, n., p.507-512, 1974.

WUTKE, E. B.; ARÉVALO, R. A. Adubação verde com leguminosas no rendimento da cana-de-açúcar e no manejo de plantas infestantes. Campinas: Instituto Agronômico, 2006. 28p. (Boletim Técnico IAC, 1985). 